\title{
Optical trapping of microparticles and yeast cells at ultra-low intensity by intracavity nonlinear feedback forces
}

\author{
Fatemeh Kalantarifard $^{\mathrm{a}}$, Parviz Elahi ${ }^{\mathrm{a}}$, Ghaith Makey ${ }^{\mathrm{b}}$, Bürçin Ünlü ${ }^{\mathrm{a}}$, Onofrio M. Maragòc ${ }^{\mathrm{c}}$ F. \\ Ömer Ilday ${ }^{\mathrm{b}, \mathrm{d}, \mathrm{e}}$, and Giovanni Volpe ${ }^{\mathrm{f}}$ \\ aDepartment of Physics, Boğaziçi University, 34342, Istanbul, Turkey. \\ ${ }^{\mathrm{b}}$ Department of Physics, Bilkent University, 06800, Ankara, Turkey. \\ ${ }^{\mathrm{c}} \mathrm{CNR}-\mathrm{IPCF}$, Istituto per i Processi Chimico-Fisici, 98158 Messina, Italy. \\ dDepartment of Electrical and Electronics Engineering, Bilkent University, 06800, Ankara, \\ Turkey. \\ eUNAM - National Nanotechnology Research Center and Institude of Material Science and \\ Nanotechnology, Bilkent University, 06800, Ankara, Turkey. \\ fDepartment of Physics, University of Gothenburg, 41296 Gothenburg, Sweden.
}

\begin{abstract}
In standard optical tweezers optical forces arise from the interaction of a tightly focused laser beam with a microscopic particle. The particle is always outside the laser cavity and the incoming beam is not affected by the particle position. Here we describe an optical trapping scheme inside the cavity of a fiber laser where the laser operation is nonlinearly influenced by the displacement of trapped particle and there is a coupling between laser operation to the motion of the trapped particle and this can dramatically enhances optical tweezers action and gives rise to nonlinear feedback forces. This scheme operates using an aspheric lens at low numerical aperture $(\mathrm{NA}=0.125)$, NIR wavelength $(\lambda=1030 \mathrm{~nm})$, and very low average power which results in about two orders of magnitude reduction in exposure to laser intensity compared to standard optical tweezers. Ultra-low intensity at our wavelength can grant a safe, temperature-controlled environment, away from surfaces for microfuidics manipulation of biosamples that are sensitive to light intensity. As the main advantage of our approach and highly relevant application, we observed that we can trap single yeast cells at a very low power, corresponding to an intensity of $0.036 \mathrm{~mW} \mathrm{~mm}^{-2}$, that is more than a tenfold less intensity than standard techniques reported in the literature.
\end{abstract}

Keywords: Optical tweezers, Optical trapping, Intracavity optical trapping, Fiber laser

\section{INTRODUCTION}

Since the pioneering work by A. Ashkin, ${ }^{1,2}$ optical tweezers ${ }^{3}$ (OT) have been used as a powerful technique for capturing and manipulating sub-micron particles in various research fields from soft-matter and spectroscopy ${ }^{4}$ to biology ${ }^{5}$ and nanoscience. ${ }^{6}$

Standard OT consists of a single beam with a Gaussian profile which is focused by a high numerical aperture typically, NA> 1.2 water or oil immersion microscope objective to provide strong enough gradient forces being able to trap particles stably. On a single particle two kinds of forces are usually identified. The scattering force, which is due to the radiation pressure of the light beam and acts in the direction of propagation of the beam, and The gradient force, which pushes a dielectric particle (whose refractive index is higher than the embedding medium) towards the high-intensity spot of an optical field. A main concern is that the interaction of trapping laser beam on biological samples induces a damage on the specimen which is mainly due to light absorption of the sample. ${ }^{7}$ Therefore there are important factors that must be considered, to secure the viability of cell trapping, such as wavelength, spot size of the focused laser beam and its power at the sample plane. Thus, it is of crucial

Further author information: (Fatemeh Kalantarifard)

E-mail: fatemeh.kalantarifard@boun.edu.tr

Optical Trapping and Optical Micromanipulation XVII, edited by Kishan Dholakia,

Gabriel C. Spalding, Proc. of SPIE Vol. 11463, 1146309 - ( 2020 SPIE

CCC code: $0277-786 \mathrm{X} / 20 / \$ 21 \cdot$ doi: $10.1117 / 12.2568420$

Proc. of SPIE Vol. 11463 1146309-1 
importance to look for new configurations with low NA with the capability of creating 3-D confinement at low intensity. In this work, we present a new approach for three dimensional trapping of micro particles and living cells by a feedback method. Dynamical systems based on the interchange of feedback mechanism are prevasive in science and technology including from photonics such as wavefront shaping and polarization control. Feedback methods have also a key importance in recent experiments for laser cooling of levitated particles in vacuum. ${ }^{8}$

Here we focus on the nonlinear feedback mechanism arising from implementing the trapping optics inside a fiber laser cavity and this creates a coupling between the laser signal and trapped particle. ${ }^{9}$ The result of nonlinear feedback is to hold micro objects stably due to a power self-regulation occurring he optomechanical coupling between the laser cavity and the trap. We perform a simulation to describe intracavity optical tweezers (IcOT) and demonstrate a home-built IcOT to trap a living cell and different kinds of micro particles. In this configuration, we employed a common lens with low numerical aperture $(\mathrm{NA}=0.125)$ to focus a Gaussian beam inside the laser cavity. This enables a reduction of the average light intensity employed for a given trap stiffness minimizing the possible photodamage at the sample. In contrast, in the standard OT case at low NA and corresponding low power, particles are pushed away from the trap region and when compared with a standard OT with high NA $(\mathrm{NA}=1.3)$, the trap stiffness per unit intensity $(k / I)$ of the IcOT is two order of magnitudes higher.

\section{METHODS}

The working principle of intracavity optical trapping is shown in Figure 1. When there is no particle in the trap region (Fig. 1a), the intracavity laser power $P$ is high, and as a result the particle is attracted towards the center of the trap. When a particle is trapped (Fig.1b), cavity losses of the cavity by the particle are maximum because the particle scatters light out of the cavity and increases losses. When the particle fluctuates away from the focal region (Fig.1c), its scattering decreases, and trap power increases.

Analytical toy model We developed a toy model that permits an analytic solution and to simplify the solution, our discussion on dynamics is restricted in the horizontal plane. The results can readily be generalized to the vertical direction with the inclusion of gravity. The variation of the lateral displacement of particle from the center of the trap is given by an overdamped Langevin equation: ${ }^{3}$

$$
\dot{r}(t)=-\frac{k}{\gamma} r(t)+\sqrt{2 D} \xi(t),
$$

where $r(t)$ is the particle displacement from its equilibrium position, $k$ is the trap stiffness, $\gamma$ is the particle friction coefficient, $D$ is the particle diffusion coefficient, and $\xi(t)$ is a stochastic term corresponding to white noise with zero mean and unit power. The variance of the particle position in the trap is given by

$$
\sigma_{\mathrm{r}, \mathrm{OT}}^{2}=\frac{k_{\mathrm{B}} T}{k},
$$

where $k_{\mathrm{B}}$ is the Boltzmann constant and $T$ is the ambient temperature. The trap stiffness is proportional to the laser power,

$$
k=\kappa_{\mathrm{P}} P,
$$

where $\kappa_{\mathrm{P}}$ is a constant determined by the geometrical and optical properties of the setup and the sample, but independent of the laser power. For standard optical tweezers, $P$ and $k$ are independent of $r$ but in the intracavity optical trapping scheme, the difference is that the optically trapped particle acts as a part of the cavity and it's position modulates the loss of the cavity. ${ }^{9}$ We describe this coupling using a known model for laser dynamics by H. Haken. ${ }^{10}$ The net gain of the laser is negative for particle displacements smaller than a specific amount, so that the laser remains off and when the particle reaches beyond $r_{\mathrm{L}}$, the laser power turns on. Therefore, the stationary value of the laser power is ${ }^{9}$

$$
P(r)= \begin{cases}0 & r \leq r_{\mathrm{L}} \\ P_{0}\left(\frac{r^{2}}{r_{\mathrm{L}}^{2}}-1\right) & r>r_{\mathrm{L}}\end{cases}
$$



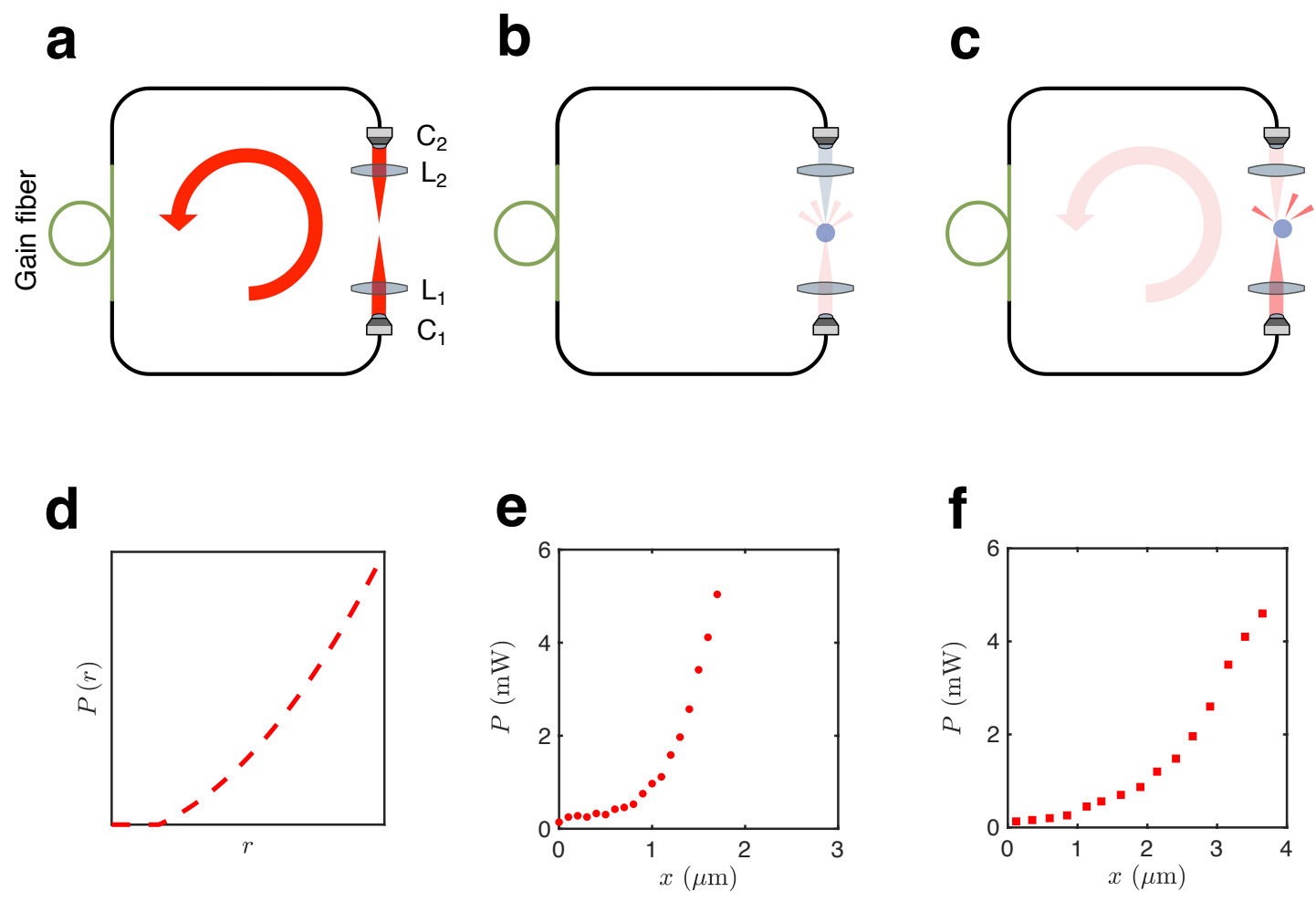

Figure 1. Schematic of working principle. The trapping optics is inserted within the ring cavity of a fiber laser so that the position of the optically trapped particle can influence the operation of the laser. Without particle (a), laser feedback power is above threshold, and so the particle is attracted to the trap region. When a particle is trapped (b), reduces the feedback by scattering most of the light out of the cavity and it increases again when the particle tries to escape (c). Therefore, intracavity laser power depends on the positions of the particle and it is shown by analytical calculation (Eq. 4) (d). The dependency relation is then confirmed by numerical simulation (e) and experimental results (f) obtained for a $4.9 \mu \mathrm{m}$ diameter polystyrene particle moved along $x$ direction in the trap plane. Adapted from reference [9].

where $P_{0}$ is a constant.

Using Eq. (3), the restoring force is $F(r)=-k r=-\kappa_{\mathrm{P}} P(r) r$, which is now non-harmonic because the trap stiffness depends on $r$ in the intracavity optical trapping scheme. this positional dependency constitutes the nonlinear feedback force. Integrating the force, we obtain the corresponding trap potential $U(r)=-\int_{0}^{r} F(x) \mathrm{d} x$, and using the Boltzmann factor, the probability density of the particle position becomes ${ }^{9}$

$$
\rho(r)=\rho_{0} e^{-\frac{U(r)}{k_{\mathrm{B}} T}}= \begin{cases}\rho_{0} & r \leq r_{\mathrm{L}} \\ \rho_{0} e^{-a r^{4}+b r^{2}-\frac{1}{2} b r_{\mathrm{L}}^{2}} & r>r_{\mathrm{L}}\end{cases}
$$

where $a=\frac{P_{0} \kappa_{\mathrm{P}}}{4 r_{\mathrm{L}}^{2} k_{\mathrm{B}} T}, b=\frac{P_{0} \kappa_{\mathrm{P}}}{2 k_{\mathrm{B}} T}$, and $\rho_{0}$ is the normalization factor. This model can be solved exactly, obtaining expressions for the variance of the particle position, $\sigma_{r}^{2}=\int_{0}^{\infty} r^{2} \rho(r) \mathrm{d} r$, and the average laser power to which the particle is exposed, $P_{\text {ave }}=\int_{0}^{\infty} P(r) \rho(r) \mathrm{d} r$.

Numerical simulation We now present a series of numerical simulations based on an extended theoretical model, including highly realistic descriptions of the laser dynamics, optical losses incurred by the particle, and the particle's Brownian motion in order to gain a quantitative understanding of the dynamics of intracavity optical trapping and to guide the experiments. The dynamics of a trapped particle in proximity of its equilibrium position is governed by the interplay between the optical force, $\mathbf{F}_{\text {trap }}[\mathbf{r}(t), P(\mathbf{r}(t))]$, viscous drag, thermal fluctuations, and gravity, $\mathbf{F}_{g}=\left(\rho_{p}-\rho_{m}\right) V g \hat{z}$, that depends on the densities of particle, $\rho_{p}$, and medium, $\rho_{m}$, 
particle volume, $V$, and gravity acceleration, $g$. One should notice that, in the intracavity OT laser power that creates the optical forces and is responsible for trapping, is not constant but changes over time according to the displacement of the trapped particle. This instantaneous power is calculated using laser rate equations for each time step (see method). At low Reynolds number it can be described by an overdamped Langevin equation: ${ }^{3}$

$$
\frac{d}{d t} \mathbf{r}(t)=\frac{\mathbf{F}_{\text {trap }}[\mathbf{r}(t), P(\mathbf{r}(t))]+\mathbf{F}_{g}}{\gamma}+\sqrt{2 D} \mathbf{W}(t)
$$

where $\mathbf{r}(t)$ is the particle position, $\gamma=3 \pi d \eta$ is its friction coefficient that depends on particle diameter, $d$, and medium viscosity, $\eta$. The noise terms are related to particle diffusion, $D=k_{B} T / \gamma$, that depends on temperature, $T$, and Boltzmann constant, $k_{B}$, and $\mathbf{W}(t)$ is a vector of independent white noise describing the time evolution of the thermal fluctuations in 3-D. To simulate the optical trapping force, geometrical optics approximation is applied. In the geometrical optics approach, the incoming laser beam is decomposed into a set of optical rays, which are then focused by the lens or objective. As the rays reach the particle, they get partially reflected and partially transmitted. The direction of the reflected and transmitted rays are different from those of the incoming rays. This change of direction entails a change of momentum and, because of the action-reaction law, a force acts on the sphere. If $n_{p}>n_{m}$, these optical forces tend to pull the sphere towards the focal region to create an equilibrium position. As the scattered rays reach the second lens or objective, they are collected and projected onto the back-focal plane input of the fiber. The laser dynamics is modeled using standard power rate equations. ${ }^{11}$ This is a highly realistic model considering the gain saturation and the fact that the characteristic timescale for particle Brownian motion is in the milliseconds compared with the laser dynamics which is about nanoseconds. The laser is always at its steady state and its power is calculated using the laser rate equations and the loss corresponding to the particle position. The particle position is the result of total force exerted on the trapped particle and it is obtained from the power. This coupling between laser power, force and particle position is shown in the block diagram of the simulation in 2a.

Experiment The schematic of the IcOT experimental setup is shown in Fig. 2b. This is based on a continuouswave $(\mathrm{CW})$ Yb-doped fiber laser system operating at $1030 \mathrm{~nm}$. Such a laser system is realized to operate with a power up to several hundreds milliwatts with a power fluctuation much less than $0.01 \%$. The trapping optics is implemented in the cavity so that the displacements of a trapped particle can influence directly the optical properties of the cavity. The laser beam is directed upward and the trap is achieved by a lens with actual numerical aperture $\mathrm{NA}=w / f=0.125$, where $w$ is the beam waist at the lens and $f$ is the lens focal length. The laser power in the cavity depends on the loss of all components including the trapped particle. Since the trapped particle position changes due to thermal fluctuations, optical forces and gravity, the loss due to the particle keeps changing and, as a result, the feedback and power of the laser beam responsible for trapping is influenced according to the particle position. Thus, as a consequence of this optomechanical coupling, a correlation between laser power and particle position builds up. We performed intracavity of trapping two different types of spherical particles, silica and polystyrene beads. Also, we were able to trapped yeast cells at very low intensity compared with standard optical tweezers using high NA objectives. Note that the these micro-objects in the standard OT configuration with the same lens (low NA), cannot stay stable in the axial direction because of the weakness of axial optical trapping forces.

\section{RESULTS AND DISCUSSIONS}

Simulation results In Fig. 3 we show the simulations' results for a $4.9-\mu$ m-diameter polystyrene particle held in an intracavity optical trap. When the particle is in the center of the trap, a significant part of the light is scattered out of the collector lens, which leads to a low laser power (position i in Fig. 3a). When the particle moves away from the trap along the radial direction, this leads to an increase of the laser power that reaches the collector lens (position ii in Fig. 3a) and, therefore, to an increased restoring force pulling back the particle. Similarly, when the particle moves down along the axial direction, there is an increase of the collected power (position iii in Fig. 3a) and an increase of the scattering force that pushes the particle up. Finally, when the particle moves up along the axial direction, the rays are scattered away from the collector lens (position iv in 


\section{a}
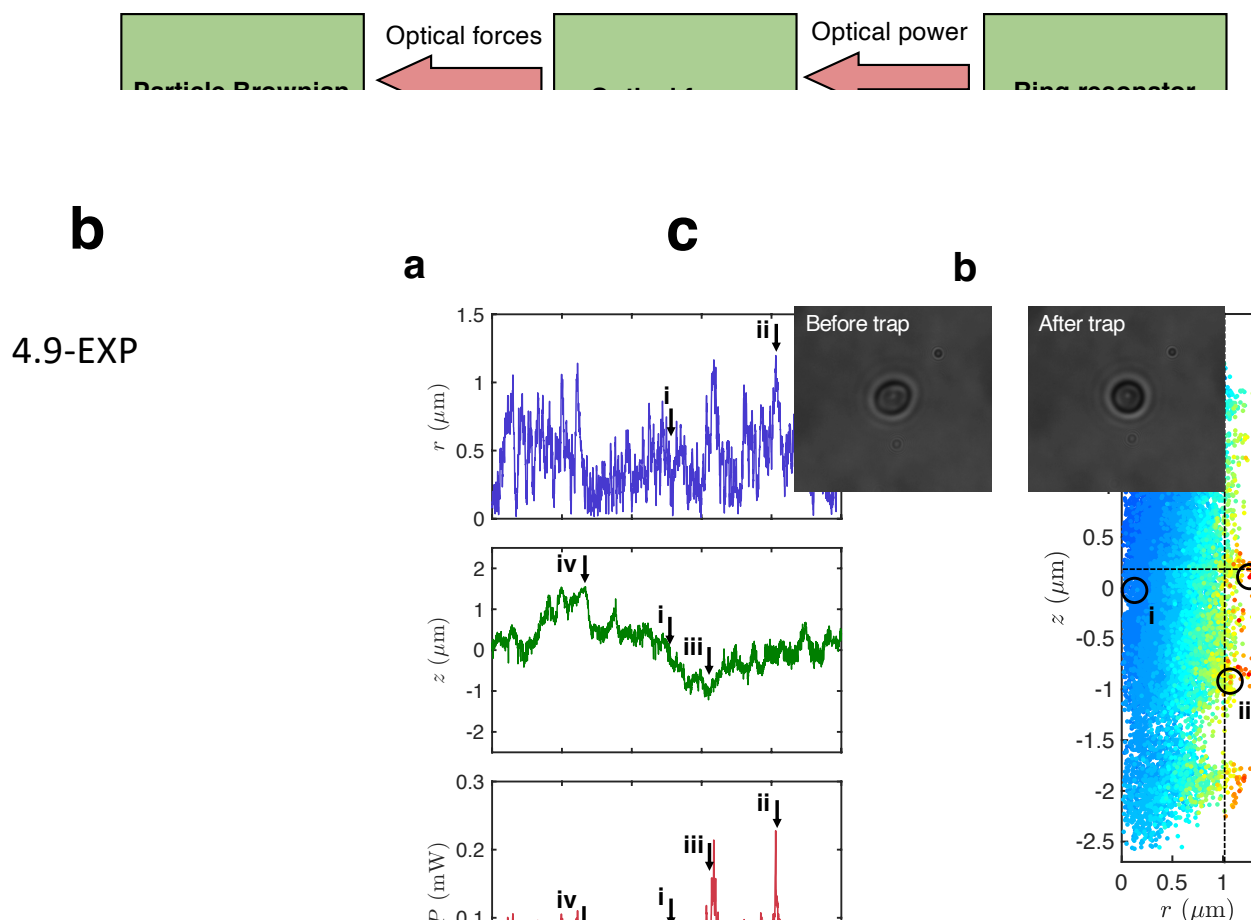

Figure 2. Schematic of numerical simulation and experimental setup of intracavity optical tweezers. Block diagram of the simulation consists of three major software packages: Ring resonator simulation ; Optical forces and scattering of the particle simulation; and Brownian motion of the particle simulation. The three packages share information as shown by the arrows: Optical force simulation receives in input the information on the optical losses from particle Brownian motion; Force calculation receives the information on the optical power from ring resonator and on the position of the particle from Brownian motion; Brownian motion simulation receives the information on the optical forces from the optical force simulation (a). Schematic of the experimental setup of intracavity optical tweezers (b). The cavity includes a diode-pumped Yb-doped fiber laser and the optical trapping setup, where WDM: wavelength division multiplexer. A living yeast cell in the intracavity optical tweezers before and after trap (c). The average power at the cell is $0.47 \mathrm{~mW}$ and calculated laser intensity on the sample is $0.036 \frac{\mathrm{mW}}{\mu^{2}}$.

Fig. 3a) and this decreases the laser power letting the particle fall back towards the center of the trap. In addition, we have analyzed the effective optical potential generated by the intracavity optical trap using the trajectories of an optically trapped particle obtained from simulations (Fig. 3b-g) and experiments (Fig. 4bg). We have analyzed these trajectories using three standard methods, potential analysis, correlation analysis and power spectrum analysis, which are based on the Langevin description of a Brownian particle in thermal equilibrium and give a direct calibration of the trap stiffness of the effective optical potential. ${ }^{3,12}$

The results of all analyses are consistent with an effective harmonic confinement for small displacements around the equilibrium position and short times. Thus, we can measure the associated meaningful transverse and axial force constants. However, we remark that deviations emerge for large displacements and long times.

Experimental results Fig. 4 illustrates the results obtained by trapping a $4.9-\mu \mathrm{m}$-diameter polystyrene particle. Fig. 4 a shows the time evolution of the particle radial position $r$, its axial position $z$, and the laser power $P$. Fig. 4a shows how the laser power depends on the radial and axial particle position, which is also in agreement with the results of the simulations presented in Fig. 3a. Along the radial direction, the laser power increases when $r$ increases, with enough restoring force that pushes the particle back towards the center of the trap. Along the axial direction, the laser power decreases when the particle moves upwards and increases when the particle 
a
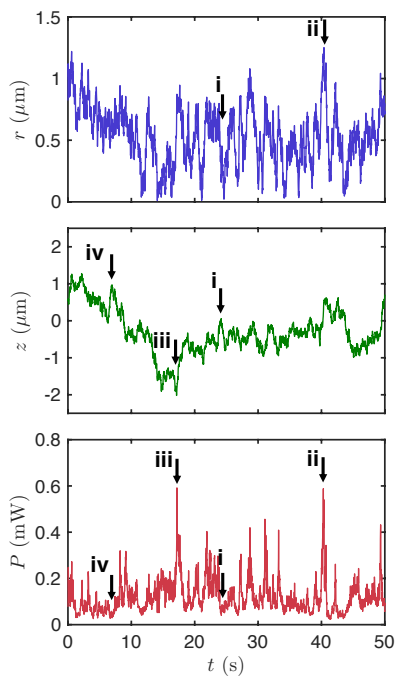

b
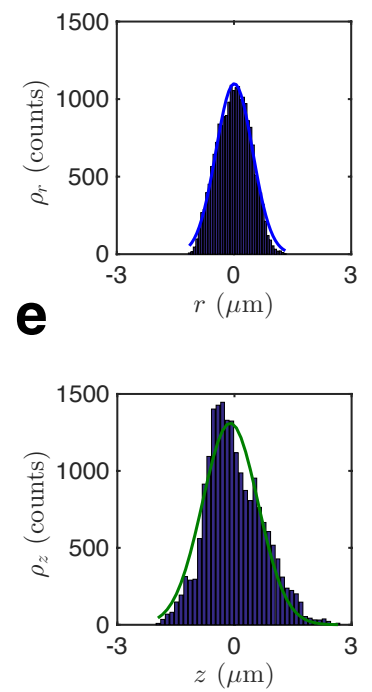

C
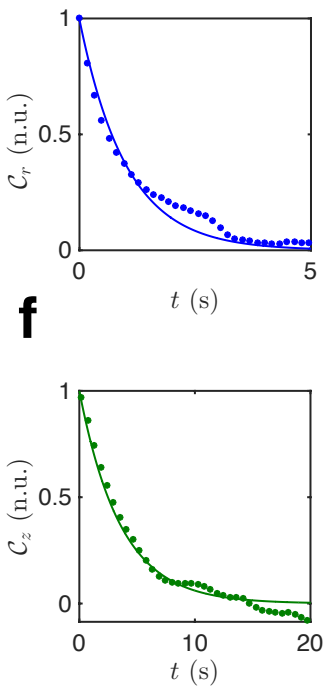

d
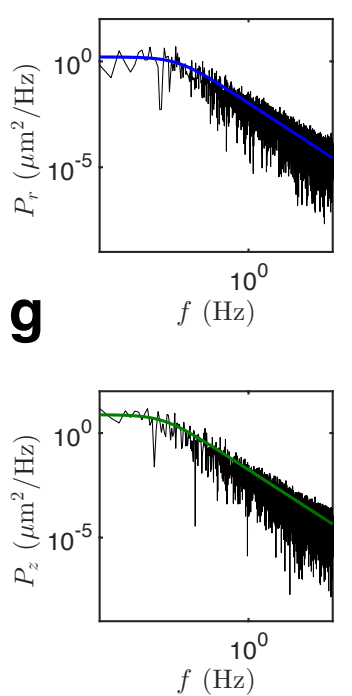

Figure 3. Simulation results. Radial $(r)$ and axial $(z)$ particle position, and corresponding laser power $(P)$ obtained from the simulation of the motion of a 4.9 - $\mu$ m-diameter polystyrene particle trapped in the intracavity optical trap (a). when the particle is at the center of the trap (equilibrium position in the trap (i) when it is displaced in the radial direction (ii) when it is displaced along the axial direction downwards (iii) and upwards (iv). Probability distribution (b), position autocorrelation functions (ACFs) (c) and power spectral densities (PSDs) (d) along the transverse x-direction (corresponding to the results shown in a). Probability distribution, ACF and PSD along the axial z-direction (e-g). The solid lines are the best fit to the data from which we calculate the trap stiffnesses of the simulated intracavity trap for small displacements and short times, which permits us to use standard techniques for calibration. Adapted from reference $[9]$.

moves downwards, leading to enhanced scattering forces pushing the particle back to up. We have suspended polystyrene or silica microparticles in water and placed a droplet of the resulting solution in a sample chamber realized between two microscope slides separated by a para-film layer (100- $\mu$ m thickness). We recorded the motion of the particle using a CMOS camera and used digital video microscopy to track its trajectory in 3-D.

Intracavity optical trapping of yeast cells To optically manipulate living objects, as we already discussed, one of the most important factor that must be taken into account is the intensity of the laser light on the cell since it is potentially harmful causing photo damage. ${ }^{7}$ Ultra-low intensity at our NIR wavelength grants a secure environment, away from surfaces for microfluidics manipulation of biological samples. one of the mechanisms that can cause cell cycle destruction or inhibition in optical trapping is related with local heating. ${ }^{13,14}$ Some reported studies on Saccharomices cerevisiae yeast cells in near-infrared counterpropagating traps ${ }^{15}$ and standard optical tweezers have shown that while $3.5 \mathrm{~mW}$ power that corresponds to an intensity of about $0.33 \mathrm{~mW} / \mu \mathrm{m}^{2}$, is needed for 3-D trapping of a single yeast cell, phototoxicity is detected at $0.7 \mathrm{~mW}$ (corresponding to about 0.07 $\mathrm{mW} / \mu \mathrm{m}^{2}$ ) over long-term exposure time (few hours). In another comparison using standard optical tweezers ${ }^{16}$ minimum 3D trapping power, $10 \mathrm{~mW}$ (about $22 \mathrm{~mW} / \mu \mathrm{m}^{2}$ intensity), has been reported. We observed that we can 3D trap single yeast cells with about an intensity of $0.036 \mathrm{~mW} / \mu \mathrm{m}^{2}$, that is more than a tenfold less intensity than standard techniques. A trapped yeast cell and its monitored power is shown in Fig. 2c. The average power on the sample and its corresponding intensity is $0.47 \mathrm{~mW}$ and $0.036 \frac{\mathrm{mW}}{\mu^{2}}$ respectively.

At this stage we can compare experimental and theoretical results of our intracavity optical trapping setup with two standard optical trapping configurations operating with low $(\mathrm{NA}=0.125)$, and high $(\mathrm{NA}=1.3)$ numerical apertures. We first observe that for low NA, equivalent to the IcOT, the experimental and simulation results show that the trap is not strong enough in the axial direction. This is due to the poor gradient force of a low NA 
a
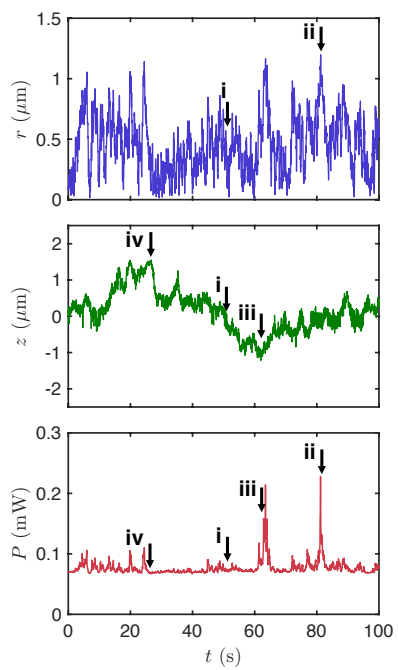

b
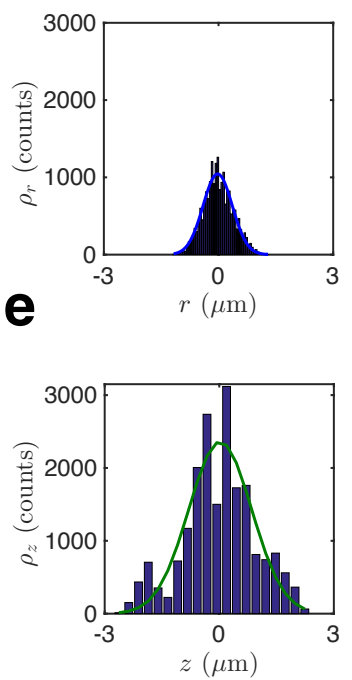

C
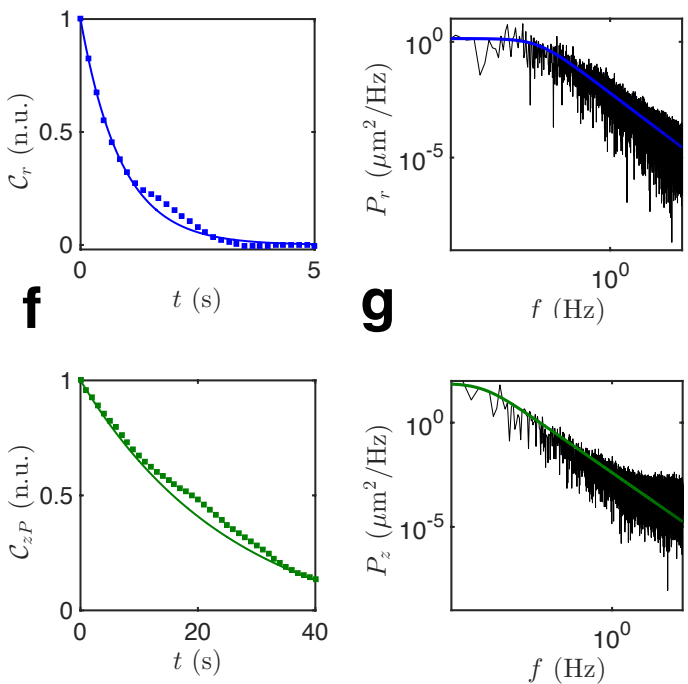

Figure 4. Experimental results. Radial $(r)$ and axial $(z)$ particle position, and corresponding laser power $(P)$ obtained from a $4.9-\mu$ m-diameter polystyrene particle experimentally trapped in the intracavity optical trap (a). when the particle is at the center of the trap (equilibrium position in the trap (i) when it is displaced in the radial direction (ii) when it is displaced along the axial direction downwards (iii) and upwards (iv). Probability distribution (b), position autocorrelation functions (ACFs) (c) and power spectral densities (PSDs) (d) along the transverse x-direction (corresponding to the results shown in a). Probability distribution, ACF and PSD along the axial z-direction (e-g). The solid lines are the best fit to the data from which we calculate the trap stiffnesses of the simulated intracavity trap for small displacements and short times, which permits us to use standard techniques for calibration. Adapted from reference [9].

lens which is not capable of providing a 3-D confinement. Fig. 5a presents the axial total force for the intracavity optical trapping case (filled circles) compared with the standard optical trapping case using the same low NA lens (open circles) for a $4 \mu \mathrm{m}$ silica particle. It shows that the intracavity axial force is one order of magnitude higher yielding a much more stable optical trap. To complete the comparison, we measured and calculated the force constants efficiency, $k_{r} / I$ and $k_{z} / I$, of our intracavity optical trapping with standard optical tweezers with high numerical aperture $(\mathrm{NA}=1.3)$ objective. Here $\left.k_{r}=\left(k_{x}+k_{y}\right) / 2\right)$ and $k_{z}$ represent the lateral and axial trap stiffness respectively, and $I$ is the intensity of the laser beam incident on the trapped particle. We trapped particles of the same kind and size with standard optical tweezers operating with high NA microscope objective $(\mathrm{NA}=1.3)$ and with laser at $976 \mathrm{~nm}$ wavelength. Then, we tracked the particles using digital video microscopy, as we did for the intracavity case, and obtained the lateral and axial trap stiffnesses. The comparison (Fig. 5b) shows that the intracavity trap efficiency (force constant per unit intensity) is consistently about two orders of magnitude higher than that for standard optical tweezers.

\section{CONCLUSIONS}

In conclusion, we have demonstrated a 3 -D optical trapping scheme at low numerical aperture $(\mathrm{NA}=0.125)$ based on an intracavity operation with a fiber laser. A thorough experimental and theoretical study has been presented showing that the threshold power of the laser increases in the presence of a trapped particle and therefore, a particle trapped at power near threshold is able to significantly decrease the laser power that goes through the sample. We have trapped yeast cells, as well as silica and polystyrene microparticles of different size. Particle tracking by digital video microscopy enabled the accurate measure of the intracavity trap force constants. The opto-mechanical coupling between particle position and laser power has been elucidated that shows how the laser power is selg-regulated by the displacement of the trapped particle. Trap force constants efficiencies $(k / I)$ have been compared with standard high NA ones. Experimental results and theory revealed that the trap efficiencies 
a

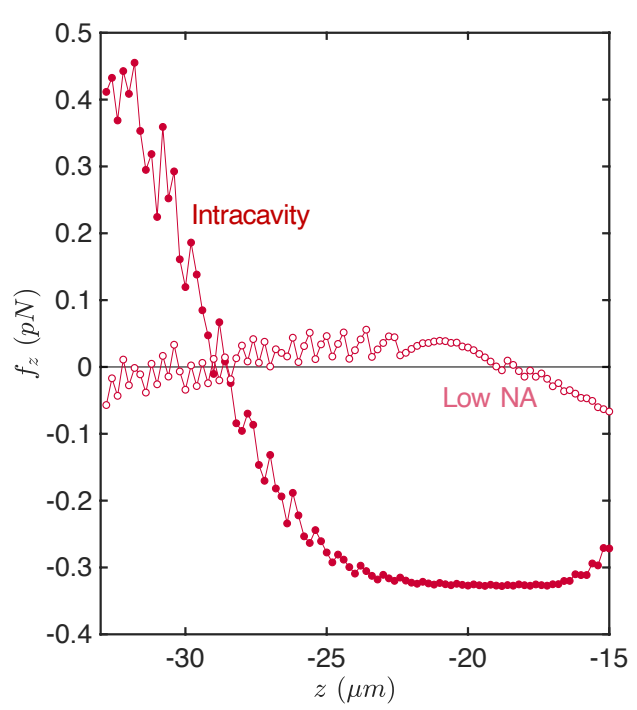

b
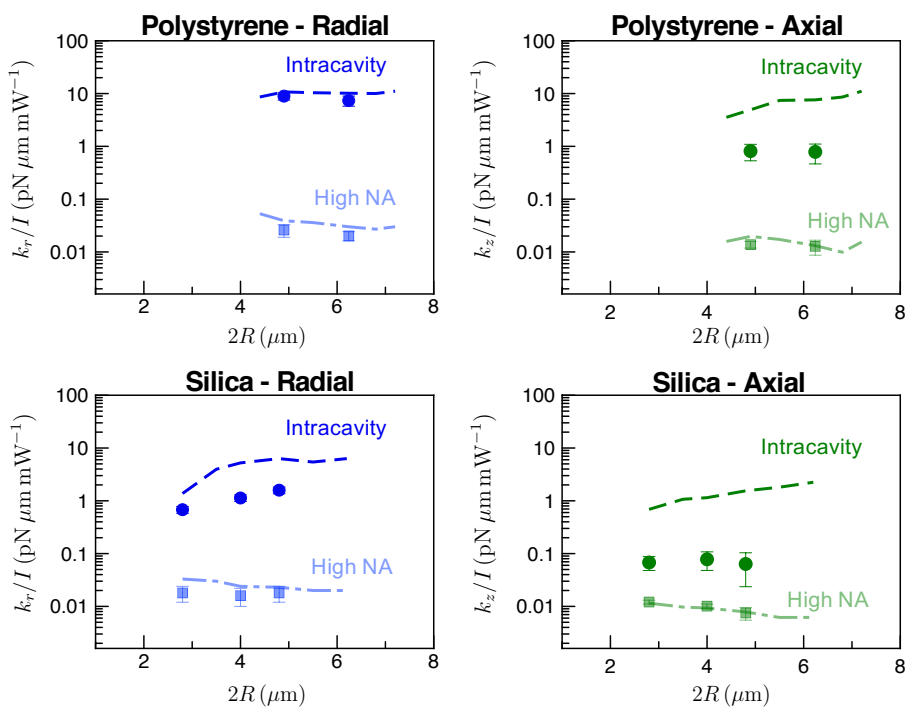

Figure 5. Comparison of an intracavity optical trap and a standard low NA and high-NA optical tweezers. Total axial force numerically calculated for a silica $4 \mu \mathrm{m}$ particle moved along axial direction in intracavity optical tweezers (filled circles) and in normal optical tweezers operating with the same NA as IcOT and at its average power (open circles) (a). Comparison of the experimentally measured trap efficiency (trap stiffness, $k_{r}$ and $k_{z}$, per unit intensity at the sample) for an intracavity optical trap (circles) and a standard high-NA optical tweezers (squares) for polystyrene and silica particles of various radii $R$. The dashed lines are the corresponding results from numerical simulations.

of intracavity optical trapping are about 2 orders of magnitude higher than the ones of the standard optical trapping. The major advantages of this ultra-low intensity scheme shines up when dealing with biosamples because biological matter is sensitive to light intensity and the typical tight focus of standard optical tweezers can have detrimental effects over cell manipulation specially for long-term. Our observation of a 3-D trapping of single yeast cells has shown an ultra-low intensity at sample that is more than a tenfold less intensity than standard techniques.

\section{REFERENCES}

[1] Ashkin, A., "Acceleration and trapping of particles by radiation pressure," Phys. Rev. Lett. 24, 156 (1970).

[2] Ashkin, A., Dziedzic, J. M. Bjorkholm, J. E., and Chu, S., "Observation of a single-beam gradient force optical trap for dielectric particles," Opt. Lett. 11, 288-290 (1986).

[3] Jones, P. H., Maragò, O. M., and Volpe, G., [Optical Tweezers: Principles and Applications], Cambridge University Press (2015).

[4] Petrov, D. V., "Raman spectroscopy of optically trapped particles," J. Opt. A. Pure. Appl. Op. 9, S139 (2007).

[5] Fazal, F. M. and Block, S. M., "Optical tweezers study life under tension," Nat. Photonics 5, 318-321 (2011).

[6] Maragò, O. M., Jones, P. H., Gucciardi, P. G., Volpe, G., and Ferrari, A. C., "Optical trapping and manipulation of nanostructures," Nat. Nanotech. 8, 807-819 (2013).

[7] Singh, G. P., Volpe, G., Creely, C. M., Grötsch, H., G., I. M., and Petrov, D., "The lag phase and g1 phase of a single yeast cell monitored by raman microspectroscopy," J. Raman Spectrosc. 37, 858-864 (2006).

[8] Gieseler, J., Deutsch, B., Quidant, R., and Novotny, L., "Subkelvin parametric feedback cooling of a lasertrapped nanoparticle," Phys. Rev. Lett. 109, 103603 (2012).

[9] Kalantarifard, F., Elahi, P., Makey, G., Maragò, O. M., Ilday, F. Ö., and Volpe, G., "Intracavity optical trapping of microscopic particles in a ring-cavity fiber laser," Nature communications 10(1), 1-11 (2019). 
[10] Haken, H., [Synergetics], Springer, 3rd ed. (2013).

[11] Kelson, I. and Hardy, A. A., "Strongly pumped fiber lasers," IEEE J. Quant. Electron. 34, 1570-1577 (1998).

[12] G. Pesce, et al., "Step-by-step guide to the realization of advanced optical tweezers," J. Opt. Soc. Am. B 32, B84-B98 (2015).

[13] Liu, Y., Cheng, D., Sonek, G., Berns, M., Chapman, C., and Tromberg, B., "Evidence for localized cell heating induced by infrared optical tweezers.," Biophysical Journal 68(5), 2137 (1995).

[14] Peterman, E. J., Gittes, F., and Schmidt, C. F., "Laser-induced heating in optical traps," Biophysical Journal 84(2), 1308-1316 (2003).

[15] Aabo, T., Perch-Nielsen, I. R., Dam, J. S., Palima, D. Z., Siegumfeldt, H., Glückstad, J., and Arneborg, N., "Effect of long-and short-term exposure to laser light at $1070 \mathrm{~nm}$ on growth of saccharomyces cerevisiae," Journal of biomedical optics 15(4), 041505 (2010).

[16] Pilát, Z., Jonáš, A., Ježek, J., and Zemánek, P., "Effects of infrared optical trapping on saccharomyces cerevisiae in a microfluidic system," Sensors 17(11), 2640 (2017). 